\title{
Marxistische Theoriearbeit in der Krise
}

\author{
Roland Atzmüller
}

Zusammenfassung Die ökonomischen aber auch politischen und ideologischen Krisen und Transformationen der kapitalistischen Gesellschaftsformationen haben marxistische Ansätze in eine fundamentale Krise gebracht. Diese erfasst ihre Vorstellungen zur Notwendigkeit, Wünschbarkeit und Möglichkeit gesellschaftlicher Transformation und zu den sie erkämpfenden sozialen AkteurInnen und deren Praxisformen. Sie macht eine grundlegende Reformulierung wesentlicher epistemologischer und gesellschaftstheoretischer Annahmen notwendig. Der Beitrag argumentiert, dass die Krise des Marxismus bei einer selbstreflexiven Auseinandersetzung mit den Produktionsbedingungen marxistischer Theoriearbeit ansetzen muss. Die Anerkennung der Unabschließbarkeit und Unvollständigkeit marxistischer Gesellschaftskritik sowie ihre Bildung im Widerstreit stellen eine zentrale Form dar, um zu vermeiden, wieder in autoritäre Formen der Politik und der Legitimation von Herrschaft einbezogen zu sein. Der umfassende Charakter der Krisentendenzen in kapitalistischen Gesellschaftsformationen und die damit verbundene Multiplikation der gesellschaftlichen Konfliktfelder erfordert weiters die Überwindung einer monistischen Lesart ihrer Dynamiken. Kapitalistische Gesellschaftsformationen als Verhältnis von Verhältnissen aufzufassen, ist notwendig, um Zusammenhänge zwischen verschiedenen sozialen Strukturen und gesellschaftlichen Macht- und Herrschaftsverhältnissen erfassen zu können. Außerdem erfordert die Veränderungsfähigkeit kapitalistischer Gesellschaftsformationen, die in der Krise sichtbar geworden ist, eine Dynamisierung des marxistischen Reproduktionskonzeptes. Dies ist notwendig, weil im wissensbasierten Kapitalismus die Dynamisierung der relativen Mehrwertproduktion die Veränderungs- und Anpassungsfähigkeit der Subjekte und ihres Arbeitsvermögens in den Mittelpunkt stellt. Erst vor dem Hintegrund derartiger Reformulierungen

\footnotetext{
R. Atzmüller $(\square)$

Institut für Soziologie, Abteilung für Gesellschaftstheorien und Sozialanalysen, Johannes Kepler Universität Linz, Altenbergerstraße 69, 4040 Linz, Österreich

E-Mail: roland.atzmueller@jku.at
} 
marxistischer Theoriearbeit kann die Vielfalt der Emanzipationsperspektiven, die in den gegenwärtigen sozialen Auseinandersetzungen sichtbar werden, in den Blick kommen.

Schlüsselwörter Krise · Marxismus · Post-/Fordismus · Gesellschaftskritik · Soziale Emanzipation

\section{Marxist theory work in crisis}

Abstract The economic as well as political and ideological crises and transformations of capitalist social formations have created a crisis for Marxist approaches. This affects their assumption about the necessity, desirability and possibility of social transformation, but also about the actors fighting them out and their activities. Thus, a far reaching reformulation of its fundamental epistemological and theoretical assumptions is necessary. This contribution argues that the crisis of Marxism demands a self-reflective engagement with conditions of Marxist theoretical work. The acknowledgement of the fundamental openness and incompleteness of Marxist social critique as well as its contested production is indispensable to avoid authoritarian forms of politics and to be implicated into the legitimation of domination again. The encompassing character of the crisis tendencies of capitalist social formations and the multiplication of fields of conflict demands to overcome a monistic interpretation of social dynamics. To conceptualise capitalist social formations as relation of relations is an indispensable precondition for the analysis of the connections between different social structures and relations of power and dominance. Furthermore, the ability of capitalist social formations to change, which has been shown by the recent crises and transformations, demands a fundamental dynamisation of the Marxist concept of reproduction. The dynamisation of relative surplus production in knowledge based capitalism moves the adaptability of the subjects and their capacity to work centre stage. Only against this background can the plurality of perspectives for emancipation and social struggles come into view for Marxist theoretical work.

Keywords Crisis $\cdot$ Marxism $\cdot$ Post-/Fordism $\cdot$ Social criticism $\cdot$ Social emancipation

\section{Einleitung}

Die Krise des Finanzmarktkapitalismus seit 2008, die daraus resultierende Staatsschuldenkrise und die Dominanz austeritätspolitischer Strategien ihrer Bearbeitung haben eine Reihe grundlegender Analysen nach sich gezogen (Lapavitsas 2013; Altvater 2010; Jessop 2015), die die Relevanz marxistischer Wissenschaft für das kritische Verständnis der Gegenwart zeigen konnten. Diese Untersuchungen entwickelten wichtige Analysen der Krise des Finanzmarktkapitalismus, aber auch der gesellschaftlichen Auswirkungen des Krisenmanagements und der sich daran entzündenden sozialen Kämpfe und machten sie als zentrale Dimension multipler Kri- 
senprozesse der Gesellschaft sichtbar (Demirović et al. 2011; Jessop 2012). Der Anspruch marxistisch orientierter Wissenschaft geht aber über die Fähigkeit zu adäquaten Analysen der Entwicklungen in Gesellschaften, in denen die kapitalistische Produktionsweise ${ }^{1}$ herrscht, hinaus. Ihr geht es vielmehr auch darum, die wissenschaftliche Kritik kapitalistischer Gesellschaftsformationen mit bestimmten Subjekten (i.e. der „ArbeiterInnenklasse“) und deren Praxisformen, die auf ihre Veränderung abzielen, zu vermitteln. Doch zwischen diesem Selbstverständnis marxistisch orientierter Wissenschaft und der politischen Realität in der Vielfachkrise kapitalistischer Gesellschaftsformationen klafft weiterhin eine Lücke. Dies zeigt sich nicht nur an der hohen Zustimmung zu austeritätspolitischen Strategien der Krisenbewältigung in großen Teilen der Bevölkerung, sondern auch darin, dass sich „Protest“ gegen die skizzierten Entwicklungen im Aufschwung rechtspopulistischer Bewegungen und Parteien äußert. Die in der Geschichte des Marxismus vielbeschworene Einheit von Theorie und Praxis bleibt daher zerrissen.

Zwar wurden in der ersten Phase der Finanzmarktkrise und den darauf folgenden staatlichen Maßnahmen zur Stabilisierung der Wirtschaft Hoffnungen laut (Altvater et al. 2010), die vielbeschworene Hegemonie des Neoliberalismus sei nun in eine Krise geraten und es würden sich Räume für Alternativen auftun. Auch kam es in vielen Ländern (etwa im Süden Europas) zu sozialen Kämpfen und der Formierung sozialer Bewegungen gegen die Durchsetzung von Sparprogrammen. Jüngst wäre auch auf durchaus überraschende wahlpolitische Mobilisierungen linksorientierter Parteien und Politiker (Bernie Sanders, Jeremy Corbyn) zu verweisen.

Trotzdem ist mehr als ein Jahrzehnt nach Ausbruch der Finanzmarktkrise weiterhin offensichtlich, dass Hoffnungen auf einen Bruch mit der Dominanz des Neoliberalismus zumindest naiv waren. Angesichts der mitunter als Radikalisierung neoliberalen Krisenmanagements analysierten Durchsetzung ,,permanenter Austerität" (Jessop 2016) und des Aufschwungs rechtspopulistischer Bewegungen in vielen Ländern auch und gerade des globalen Nordens muss man daher m. E. von einer fortgesetzten Krise des Marxismus sprechen. Bei dieser Einschätzung geht es mir nicht um die Behauptung einer neuen Krise des Marxismus, um im wissenschaftlichen Feld diskurspolitisch Aufmerksamkeit zu erregen. Vielmehr möchte ich herausarbeiten, inwiefern die gegenwärtigen Entwicklungen in einen längeren Prozess der krisenhaften Problematisierung marxistisch orientierter Theoriebildung und Wissenschaft eingebettet sind.

Die Diagnose einer Krise des Marxismus ist nämlich nicht neu, sondern beschreibt vielmehr den „Stand des Marxismus“ (Demirovic et al. 2015) und seiner Entwicklungen seit der zweiten Hälfte der 1970er-Jahre (Haug 2010; Krätke 1996;

\footnotetext{
1 Karl Marx beginnt „Das Kapital“ (1972) mit folgendem Satz: „Der Reichtum der Gesellschaften, in welchen kapitalistische Produktionsweise herrscht, erscheint als eine ,ungeheure Warenansammlung ' $[\ldots]^{\text {“ }}$ (1972, S. 49). Diese Formulierung hebt zwar die zentrale Bedeutung der kapitalistischen Produktionsweise hervor, legt aber auch nahe, dass die Analyse konkreter Gesellschaftsformationen mehr umfassen muss als jene Produktionsweise, die in ihr herrscht. Ich werde im Folgenden die knappere Formulierung ,kapitalistische Gesellschaftsformation“ verwenden, mit der die historische Spezifik konkreter Gesellschaften sichtbar gemacht wird, die als widersprüchliche und stets prekäre Einheit verschiedener gesellschaftlicher Verhältnisse aufzufassen sind und in denen den kapitalistischen Produktionsverhältnissen eine zentrale, aber nicht determinierende Bedeutung zukommt (Jessop 1990).
} 
Kouvélakis 2008). Der marxistische Philosoph Louis Althusser (1978) hatte bereits in dieser Zeit das Aufbrechen der Krise des Marxismus² diagnostiziert.

Bei der Einordnung der gegenwärtigen Ausprägungen der Krise des Marxismus in einen längerfristigen historischen Prozess darf aber nicht übersehen werden, dass die Diagnose Althussers nicht als Prognose einer spezifischen Entwicklung in kapitalistischen Gesellschaftsformationen gedacht war. Sie fand vielmehr in einer bestimmten historischen Konjunktur statt und hatte daher in gewisser Weise einen paradoxen Charakter. Er behauptete nämlich das Aufbrechen der Krise des Marxismus in einer Phase, die von der Zunahme sozialer Kämpfe und dem Auftreten neuer sozialer Bewegungen nach 1968, einem Aufschwung kritischen Denkens an den Universitäten (Demirović 2015) sowie dem Wachstum linker Parteien und der Verankerung sozialreformerischer Regierungen in einer Reihe von Staaten (v.a.) des globalen Nordens geprägt war.

Betrachtet man die Diagnose Althussers aus der Perspektive eines längerfristigen Krisenprozesses, so werden zwei Aspekte erkennbar, die letztlich über den unmittelbaren historischen Kontext hinauswiesen. Die althussersche Krisendiagnose bezog sich auf die Probleme marxistischer Theoriearbeit und ihres Verhältnisses zur Realität realsozialistischer Regime und nahm aus heutiger Perspektive deren Unfähigkeit vorweg, ihre diktatorischen Grundlagen zu überwinden. ${ }^{3}$ Darüber hinaus bestand in der skizzierten historischen Konjunktur ihr scheinbar paradoxer Gehalt in der unausgesprochenen Prognose, dass die Probleme marxistisch orientierter Theoriearbeit und Wissenschaft und der daran orientierten politischen Praxen ihre Fähigkeit zur emanzipatorischen Transformation der kapitalistischen Gesellschaftsformationen also zu dem, was oft formelhaft als Revolution beschworen wurde - unterminieren würde.

Mit dem zeitlichen Abstand von vier Jahrzehnten zeigt sich daher, dass die „paradoxe Intervention“ Althussers, ohne dass dies intendiert war, mit längerfristigen Krisen- und Transformationsprozessen kapitalistischer Gesellschaftsformationen verbunden war. Diese haben auch die wissenschaftlichen, epistemologischen und politischen Grundlagen der überkommenen marxistisch orientierten Theoriearbeit und Wissenschaft erfasst und sie in ihrer historischen Spezifik - Wolfgang Fritz Haug hat diesbezüglich vom „fordistischen Marxismus“ (Haug 1996) gesprochen - problematisiert. Daher erscheint es mir notwendig, die fortgesetzte Krise des Marxismus, die eben nicht nur eine gegenwärtige ist, sondern geschichtlich eingebettet in den Kontext der Krisentendenzen und Veränderungen (zumindest) der Gesellschaften des globalen Nordens (Hirsch 2005; Dörre 2009; Arrighi und Moore

\footnotetext{
2 Diese Diagnose und die sich daran anschließende Debatte können übrigens kein Alleinstellungsmerkmal behaupten, ist doch auffällig, dass etwa zeitgleich Anthony Giddens eine Krise des sogenannten ,orthodoxen Konsenses“" in den Sozialwissenschaften, die er auf den Aufschwung kritischer, nicht zuletzt marxistischer, aber auch interpretativer und phänomenologischer Ansätze zurückführte, konstatierte (Giddens 1978).

3 Damit war er aber weder der Erste noch der Einzige (siehe dazu die Analysen in: van der Linden 2009).
} 
2001; Aglietta (2000 [1979]); Hardt und Negri 2000; Sum und Jessop 2015) seit den 1970er-Jahren, die auch als Krise des Fordismus ${ }^{4}$ bezeichnet wird, zu stellen.

Um die Krise des Marxismus im Kontext der Krise und Transformation kapitalistischer Gesellschaftsformationen nach dem Fordismus diskutieren zu können, möchte ich im ersten Schritt (Abschn. 2) drei Dimensionen der Problematisierung marxistischer Theoriearbeit herausarbeiten. Diese betreffen die Frage des notwendig selbstreflexiven Charakters jeglicher Auseinandersetzung mit der Krise des Marxismus sowie ihre Einbettung in ökonomische sowie politische und ideologische Veränderungsdynamiken kapitalistischer Gesellschaftsformationen. In einem zweiten Analyseschritt (Abschn. 3) möchte ich die aufgeworfenen Probleme mit Blick auf die Produktionsbedingungen marxistischer Theoriearbeit als einen offenen und umstrittenen Prozess diskutieren und exemplarisch theoretische Konsequenzen, die sich aus der Problematisierung der theoretischen und epistemologischen Grundlagen marxistischer Theoriearbeit ergeben, an ausgewählten Problemstellungen analysieren.

\section{Problemstellungen in der Krise des Marxismus}

Die Transformationen kapitalistischer Gesellschaftsformationen umfassen weitreichende und umkämpfte Veränderungen der kapitalistischen Produktionsverhältnisse auf globaler Ebene seit den 1970er-Jahren, die mit der Einführung neuer Technologien sowie Produktions- und Arbeitsprozesse verknüpft sind. Diese sind nicht zu trennen von der Durchsetzung neoliberaler und neokonservativer Regierungsprojekte und den damit verbundenen Rekonfigurationen des Verhältnisses von Ökonomie und ihren staatlichen/politischen Regulationen. Die grundlegende Reorganisation der gesellschaftlichen Arbeitsteilungen und Ungleichheitsverhältnisse im Kontext von Bildungsexpansion, Feminisierung der Arbeitswelt und Veränderungen der Familienstrukturen sowie Migrationsprozessen, die von den skizzierten politischen und ökonomischen Veränderungen weiter vorangetrieben wurden, macht außerdem deutlich, dass die Transformationsprozesse die kapitalistischen Gesellschaftsformationen in ihrer Gesamtheit erfasst haben. Die Finanzialisierungstendenzen der Akkumulation und ihre Krise seit 2008 sowie die Dominanz austeritätsorientierter Formen ihrer Bearbeitung und der Aufschwung rechtspopulistischer Bewegungen stellen vor diesem Hintergrund aktuelle Erscheinungsformen der fortgesetzten Transformationskrise dar (Streeck 2013).

Die skizzierten Krisen- und Transformationsprozesse der kapitalistischen Gesellschaftsformationen haben m. E. die traditionellen Analysehorizonte und Kritikstrategien marxistischer Ansätze, die die in der fordistischen Schwerindustrie formierte (vorwiegend männliche) Arbeiterklasse und deren (angenommene) Interessen ins Zentrum stellten und z.B. die Entwicklungen des Kapitalismus aus Perspektive

\footnotetext{
4 Mit dem Begriff „Fordismus“ werden die national unterschiedlichen Ausprägungen des nach 1945 dominanten sozio-ökonomischen Entwicklungsmodells erfasst, das ab den 1970er-Jahren in eine grundlegende Krise geraten ist.
} 
einer zunehmenden Vertiefung seiner Widersprüche konzipierten, ${ }^{5}$ und damit die daraus folgenden Vorstellungen politischer Dynamiken (revolutionäre Überwindung des Kapitalismus) historisch überholt. Daraus ergeben sich folgende Problemstellungen für die Erneuerung marxistischer Theoriearbeit.

a. Wenn man die Entwicklungen der Krise des Marxismus aus einer längerfristigen Perspektive zu analysieren versucht, wird klar, dass diese nicht von den Transformationen der kapitalistischen Gesellschaftsformationen seit den 1970er-Jahren und den daraus resultierenden Herausforderungen seiner Reformulierung und Aktualisierung getrennt werden können. Die dauerhafte Bedeutung der paradoxen Intervention Althussers, die aus ihrem unmittelbaren historischen Kontext herrührte, liegt daher in ihrer Betonung des notwendig selbstreflexiven Gehalts der Problematisierung der wissenschaftlichen, epistemologischen und politischen Grundlagen marxistisch orientierter Theoriearbeit im Kontext gesellschaftlicher Krisen und Transformationen.

Der selbstreflexive Kern der althusserschen (1978) Krisendiagnose, der m. E. auch heute noch Gültigkeit hat, bestand erstens in der Feststellung, dass der von marxistischen Theorien behauptete Zusammenhang zwischen der kritischen Analyse kapitalistischer Gesellschaftsformationen und bestimmten sozialen AkteurInnen (wie etwa Gewerkschaften, sozialistischen/kommunistischen Parteien, dem „Proletariat"“) und dem Anspruch einer emanzipatorischen Veränderung der Gesellschaft zerrissen sei. Damit wurde deutlich, dass zwischen marxistisch orientierten wissenschaftlichen Debatten und den konkreten und komplexen sozialen Konflikten und Bewegungen zunehmend eine Lücke klaffte.

Das zweite Problem, das auch nach dem Zusammenbruch des realen Sozialismus nicht verschwunden ist, verortete Althusser in der Frage, inwieweit der Marxismus als spezifische Praxis transformatorischer Gesellschaftsanalyse und -kritik erneut „einbezogen“ (ebd., S. 57) sein könnte in Gewalt und Autoritarismus (Demirović 2008), die in seinem Namen und mit der Legitimation, für die Zukunft eine allgemeine Verbesserung des Lebens hervorzubringen, ausgeübt werden. Gerade weil sich marxistisch orientierte Theoriearbeit nicht auf die (kritische) Analyse der Gesellschaft beschränkt, sondern beansprucht, sich auf die sozialen Auseinandersetzungen um ihre Veränderung und die sie anstrebenden AkteurInnen zu beziehen, folgt daraus die Frage, ob sie ihre grundlegenden Analyse- und Kritikstrategien so reformulieren/-konstruieren kann, dass sie sich einer herrschaftsförmigen Aneignung widersetzen. Dies berührt die Produktionsbedingungen marxistischer Theorie und die darüber konstituierten theoretischen Verhältnisse und ihre Beziehungen zu den sozialen Auseinandersetzungen in den Gesellschaftsformationen an zentralen Punkten.

\footnotetext{
5 So wurde in eher orthodoxen marxistischen Ansätzen der Kapitalismus der zweiten Hälfte des 20. Jahrhunderts als Monopolkapitalismus bestimmt. Dieser Begriff beruht auf bestimmten von Marx im „Kapital“ analysierten Tendenzen der kapitalistischen Akkumulation (Konzentration) und sollte die Grenzen kapitalistischer Entwicklungsdynamiken aufzeigen. Auch der Begriff des „Spätkapitalismus“, wie er eher im Kontext der Kritischen Theorie von Adorno (2006) entwickelt wurde, deutete in gewisser Weise an, dass der Kapitalismus den Zenit seiner Entwicklungsdynamik überschritten habe und seine Überwindung längst möglich und notwendig sei.
} 
b. Die zweite Dimension betrifft die für (zumindest manche) marxistische Ansätze überraschende Veränderungsfähigkeit der kapitalistischen Gesellschaftsformationen und ihre Fähigkeit, gesellschaftliche Krisen zu überwinden, die sich seit den 1970er-Jahren auf sehr spezifische Weise manifestierte und die Annahme einer auf ihrer Überwindung hinsteuernden Vertiefung ihrer Widersprüche konterkarierte. Spätestens mit der Etablierung neoliberaler und neokonservativer Regierungsprojekte Anfang der 1980er-Jahre, die die grundlegende Transformation der Produktionsverhältnisse und der Akkumulationsprozesse sowie der damit verbundenen (keynesianischen) Wohlfahrtsstaaten auf ihre Fahne hefteten, standen marxistisch orientierte Analysen vor dem Problem, nicht mehr die als unausweichlich angenommene Krise und Überwindung des Kapitalismus erklären zu müssen, sondern seine Transformation und Erneuerung.

Die krisenhaften Transformationen der kapitalistischen Produktionsweise seit den 1970er-Jahren mussten daher nun auch als Phase radikaler Innovationen der Produktivkräfte (Informations- und Kommunikationstechnologien, Biotechnologie usw.) (Atzmüller 2014, S. 41 ff.; Perez 2012; Freeman und Soete 1997), weitreichender Transformationen der Akkumulationsprozesse (Übergang zum Finanzmarktkapitalismus, Globalisierung) (Altvater und Mahnkopf 1996; Lapavitsas 2013) sowie forcierter Inwertsetzungsprozesse nichtkapitalisierter gesellschaftlicher Bereiche auf globaler Ebene (Landnahme) verstanden werden (Dörre 2013).

Dabei stellte es sich als ein wesentlicher Aspekt der Ablösung der fordistischen Entwicklungsmodelle dar, dass die Umwälzung der Produktivkräfte insbesondere in den kapitalistischen Zentren des globalen Nordens auch das Arbeitsvermögen der Ware Arbeitskraft auf spezifische Art und Weise erfasste (Dörre et al. 2012). Die (permanente) Anpassung und Neuzusammensetzung des Arbeitsvermögens im Kontext einer sich durchsetzenden humankapitalzentrierten Regulation der Lohnverhältnisse wird zum Gegenstand veränderter und spezifischer Regierungstechnologien, wie dies etwa in den Debatten zur wissensbasierten Ökonomie (Jessop 2003, 2016) sichtbar gemacht worden ist.

c. Die skizzierten Transformationen sind drittens nicht zu trennen von den Veränderungen auf Ebene politischer und ideologischer Vergesellschaftungsprozesse, die auf der Durchsetzung neoliberaler und neokonservativer Hegemonieprojekte beruhten. Diese haben sich in den letzten Jahrzehnten als fähig erwiesen, die gesellschaftlichen Entwicklungen und Krisen aus einer auf die Dynamisierung der Akkumulation abzielenden Perspektive zu interpretieren und daraus überaus flexible, aber durchaus kohärente politische Programmatiken zu entwickeln, Erstere zu bearbeiten, die Veränderung kapitalistischer Gesellschaftsformationen voranzutreiben und dafür wahlpolitischen Zuspruch in allen (also auch proletarischen) sozialen Schichten zu erhalten.

Die unter neoliberaler Dominanz vorangetriebenen Transformationen der kapitalistischen Gesellschaftsformationen erwiesen sich daher als eine ,passive Revolution“ (Gramsci 1991), da bestimmte Momente des popularen Protests gegen den Wohlfahrtsstaat fordistischer Prägung (etwa in Bezug auf bürokratische Kontrollmechanismen) und andere, bevormundende gesellschaftliche Strukturen von den neoliberalen Regierungsprojekten kooptiert und zum Motor gesellschaftlicher 
Veränderung werden konnten (Boltanski und Chiapello 2006). Durch die partielle Einbeziehung von Momenten gesellschaftlichen Protests nach 1968 gelang es neoliberalen Hegemonieprojekten, die Umwälzung sozialer Hierarchien in den letzten Jahrzehnten mit den gesellschaftlichen Krisentendenzen in verschiedenen gesellschaftlichen Feldern (Geschlechterverhältnisse, Migration, Sexualität usw.), die quer zu den Klassenverhältnissen liegen, zu artikulieren und als negative Auswirkungen der sozialen und politischen Entwicklungen seit $1968 \mathrm{zu}$ denunzieren (Hall 1986; Demirović 2018). In den skizzierten Dynamiken der ,passiven“ Revolutionierung der kapitalistischen Gesellschaftsformationen wurde das Verhältnis der neu formierten sozialen Kräfte zum überkommenen politischen und ideologischen Institutionengefüge verändert. Populistische Momente politischer Mobilisierung wurden zu einer wesentlichen Dimension der krisenhaften Transformation kapitalistischer Gesellschaftsformationen, die sich eben nicht auf rechtspopulistische Bewegungen und Parteien begrenzen lassen, sondern auf grundlegende Veränderungen der politischen Prozesse und des Staates verweisen (Bruff 2013; Davidson und Saull 2017).

\section{Theoriearbeit gegen die Krise des Marxismus}

\subsection{Zur Selbstreflexivität marxistisch orientierter Theoriearbeit in der Krise}

Die selbstkritische Reflexion der Probleme marxistischer Theoriearbeit ist gerade in gesellschaftlichen Krisen- und Transformationsprozessen und den daraus folgenden Schwierigkeiten, diese adäquat zu erfassen, notwendig, da in den marxistischen Debatten auf solche Tendenzen immer wieder mit Strategien der Dogmatisierung reagiert wurde. Die dogmatische Selbstimmunisierung ihrer wissenschaftlichen und theoretischen Annahmen verunmöglicht nicht nur die kritische Analyse gesellschaftlicher Transformationsprozesse, sondern lässt marxistische Vorstellungen der Gesellschaftsveränderung zu formelhaften Beschwörungen verkommen, sodass sie politisches Handeln immer weniger begründen können. Vor diesem Hintergrund lassen sich drei Vorbedingungen für die Erneuerung marxistischer Theoriearbeit identifizieren.

Erstens hatte schon Althusser festgestellt, dass die Krise marxistisch orientierter Theoriebildung nicht damit beantwortet werden kann, eine als ursprünglich angenommene „Reinheit“ und „Vollständigkeit“ der Theorie (Althusser 1978, S. 62) wieder freizulegen, die durch bestimmte Theorie- und Praxisformen deformiert und verzerrt worden sei. Zweitens ist davon auszugehen, dass sich die marxistisch orientierte theoretische und wissenschaftliche Praxis im Konflikt und Widerstreit - also im „Handgemenge“ (Marx 1961b) - entwickelt, und zwar eben weil sie unabgeschlossen und unvollständig ist. Der theoretische Streit ist eine Bewegungsform der Gesellschaftskritik. Drittens muss die Erneuerung kritischer Gesellschaftstheorie(n) von den „Schwierigkeiten, Widersprüchen und Lücken“ (Althusser 1978, S. 62) ihrer theoretischen und wissenschaftlichen Grundlagen ausgehen. Dabei geht es nicht darum, marxistisch orientierte Wissenschaft asymptotisch an eine vollständigere Ana- 
lyse und Kritik kapitalistischer Gesellschaftsformationen heranzuführen. Vielmehr sind die „Schwierigkeiten, Widersprüche und Lücken“ stets aufs Neue und in Auseinandersetzung mit den Veränderungen der jeweiligen Gesellschaftsformationen zu identifizieren und zu analysieren.

Vor dem Hintergrund dieser Überlegungen ist daher zu betonen, dass jede neue Lektüre des marxistischen Theoriekorpus sich in ihrer historischen (und geographischen) Situiertheit reflektieren muss. Spezifische Lese- und Reformulierungsstrategien werden nämlich immer auch in Auseinandersetzung mit konkreten gesellschaftlichen Problemstellungen und vor dem Hintergrund zeitgenössischer Debatten erarbeitet und können nicht als Wiederentdeckung der Theorie in reiner Form verstanden werden. Die Krise marxistischer Theoriearbeit mit der Rekonstruktion eines angenommenen eigentlichen und ursprünglichen Gehalts des marxschen Denkens zu beantworten, läuft daher Gefahr, ein autoritäres Theorieverhältnis zu konstituieren, in dem im wahrsten Sinn des Wortes (neue) Gralshüter der reinen Lehre über die Art und Weise der Aneignung und Anwendung der Theorie sich zu urteilen berufen fühlen und so kritische Auseinandersetzungen verunmöglichen.

Eine zentrale Antwort auf diese Problemstellungen besteht in den an Marx orientierten Debatten daher darin, die historisch (und regional) spezifische Formierung marxistischer Ansätze und darauf Bezug nehmender transformativer Praxen in ihrer Unvollständigkeit und Unabgeschlossenheit konstitutiv in die Theoriearbeit einzubeziehen (Laclau und Mouffe 1991; Jessop 1990). Gerade weil marxistische Theoriearbeit auf gesellschaftliche Veränderungsprozesse bezogen ist, können ihre konkreten Formulierungsversuche und Analysen nicht beanspruchen, die Totalität der Gesellschaft vollständig zu erfassen. Vielmehr stellt die Pluralität möglicher Perspektiven ein konstitutives Merkmal marxistisch orientierter Theoriearbeit dar. Es ist daher notwendig, diese nicht durch ihre theoretische Abschließung zu verengen (siehe dazu auch: Laclau und Mouffe 1991), sondern sie offen und mit anderen Ansätzen und Erkenntnissen artikulierbar zu halten.

Diese Bestimmungen haben auch Konsequenzen für das marxistisch orientierte Verständnis gesellschaftsverändernder Praxisformen und der sie erkämpfenden Subjekte. Diese können nämlich nicht mehr exklusiv auf bestimmte AkteurInnen (das Proletariat), Organisationsformen (die Partei) oder Strategien (die Revolution) reduziert werden. Vielmehr eröffnet sich die Perspektive auf eine Vielzahl von gesellschaftsverändernden Praxisformen, sozialen Bewegungen und Subjekten (Hirsch 1990; Laclau und Mouffe 1991; Hardt und Negri 2000, 2004; Demirović 2011) (s. unten).

\subsection{Marxistische Theoriearbeit als Analyse ,vieler Bestimmungen“6}

Die traditionell vorherrschende Kritikstrategie vieler marxistisch orientierter Ansätze beruhte darauf, die Totalität kapitalistischer Gesellschaftsformationen und deren Dynamik auf einen zentralen Widerspruch, wie etwa auf den Kampf zwischen den sozialen Klassen (ArbeiterInnenklasse vs. Bourgeoisie) oder auf die angenommenen Auswirkungen der Warenform oder des Wertgesetzes auf alle sozialen Bereiche zurückzuführen, um von da aus mögliche Transformationsperspektiven zu eröffnen. Auch wenn diese Verhältnisse und Dynamiken in kapitalistischen Gesellschaftsfor- 
mationen von zentraler Bedeutung sind, liefen marxistische Ansätze dadurch immer wieder Gefahr, deren Relevanz zu vereinseitigen und gesellschaftliche Strukturen und Dynamiken auf eine Dynamik, eine Achse von Macht und Herrschaft, die alle gesellschaftlichen Bereiche durchdringt, zu reduzieren. Diese wurden dann zum Schlüssel einer grundlegenden und handlungsrelevanten Kritik gesellschaftlicher Totalität erklärt (Jessop 1990), während alle anderen gesellschaftlichen Prozesse als entweder kontingent und zufällig analysiert oder nur mit Blick auf ihre (Dys-)Funktionalität für die grundlegenden gesellschaftlichen Verhältnisse diskutiert wurden. Eine derartige Lesart marxistischer Theorie verdoppelte im wissenschaftlichen Feld die lukacsianische Problematik, wonach sich aus einer spezifischen Position in den Produktionsverhältnissen (bei Georg Lukacs eben derjenigen des Proletariats) die gesellschaftliche Totalität erschließe und dadurch der Schlüssel zur Transformation der Gesellschaft sichtbar würde. Die analytische Priorisierung einer spezifischen Widerspruchsdimension korrespondierte daher mit spezifischen Vorstellungen zu den sozialen AkteurInnen und Aktionsformen gesellschaftlicher Veränderung.

Diese Vorstellungen sind in den letzten Jahrzehnten durch eine Reihe von Entwicklungen massiv problematisiert worden, die m. E. wesentlich zur Krise marxistischer Theoriearbeit beigetragen haben. So kam es nach $1968 \mathrm{zu}$ einer Vervielfältigung sozialer Bewegungen (Feminismus, antirassistische Bewegungen, Ökologiebewegung usw.) und der damit artikulierten sozialen Auseinandersetzungen und Problemlagen. Die Multiplikation der sozialen AkteurInnen korrespondierte auch mit einer Vervielfältigung der gesellschaftlichen Bereiche, die zum Medium und Feld sozialer Kämpfe wurden und nicht mehr nur die Fabrik bzw. Arbeitsverhältnisse oder die politische Organisierung in Parteien umfassten. Dabei wurden insbesondere die verschiedenen Bereiche der sozialen Reproduktion, wie etwa die geschlechtlichen Arbeitsteilungen im Familienhaushalt und in der Sorgearbeit (Aulenbacher und Dammayr 2014), aber auch die in den wohlfahrtsstaatlichen Apparaten organisierten Formen des „kollektiven Konsums“ zu einem Feld sozialer Kämpfe. Daher erfasste die ,große“ (Boyer und Saillard 2002) oder „organische“ (Gramsci 1991) Krise der kapitalistischen Gesellschaftsformationen seit den 1970er-Jahren nicht nur die Akkumulationsprozesse und Produktionsverhältnisse bzw. deren (wohlfahrts-)staatliche Einbettung. Sie manifestierte sich vielmehr auch in unterschiedlichen, ungleichzeitigen und relativ autonomen Krisentendenzen in anderen sozialen Bereichen und Verhältnissen. Die Dominanz des Neoliberalismus kann daher nicht auf eine Ökonomisierung und Vermarktlichung aller Lebensbereiche reduziert werden.

In diesem Kontext ist es nämlich, wie oben bereits angedeutet, bemerkenswert, dass es insbesondere neoliberalen und neokonservativen Parteien und Bewegungen gelang, ihre politischen Projekte zur Wiederherstellung der Dynamiken kapitalistischer Akkumulationsprozesse mit Entwicklungen und Krisen in anderen gesellschaftlichen Bereichen (Migration, Veränderungen geschlechtlicher Arbeitsteilungen, Liberalisierung der Sexualität usw.), die sich nicht auf die Klassenstrukturen kapitalistischer Gesellschaftsformationen zurückführen lassen, zu verbinden, und so grundlegende Transformationen der kapitalistischen Gesellschaftsformationen in ihrer Gesamtheit voranzutreiben. Die skizzierte Vervielfältigung der sozialen Konfliktfelder und Krisen bzw. die daraus resultierenden Anforderungen an ihre kritische Analyse haben daher die endgültige Überwindung der skizzierten monistischen 
Lesarten der Dynamiken kapitalistischer Gesellschaftsformationen erforderlich gemacht. Dies bedeutet aber keinen Bruch mit, sondern erfordert vielmehr einen Rückgriff auf Marx. Dieser hatte nämlich in seinen (wenigen explizit) methodischen Überlegungen betont (1961a, 1961c), dass die kritische Analyse von der Mannigfaltigkeit und Komplexität konkreter Gesellschaftsformationen, die eine „Zusammenfassung vieler Bestimmungen sind“ (Marx 1961a, S. 632), ausgehen müsse.

Das Konkrete ist konkret, weil es die Zusammenfassung vieler Bestimmungen ist, also Einheit des Mannigfaltigen. Im Denken erscheint es daher als Prozeß der Zusammenfassung, als Resultat, nicht als Ausgangspunkt, obgleich es der wirkliche Ausgangspunkt und daher auch der Ausgangspunkt der Anschauung und der Vorstellung ist. (Marx 1961a, S. 632)

Folgt man diesen Überlegungen, so kann die Analyse kapitalistischer Gesellschaftsformationen nicht einfach aus der Perspektive eines als zentral angenommenen Verhältnisses (des kapitalistischen Produktionsverhältnisses) entwickelt werden. Vielmehr müssen konkrete kapitalistische Gesellschaftsformationen als Verhältnis von Verhältnissen, die sich wechselseitig überformen, sodass deren Beziehungen zueinander von zentraler Bedeutung sind und ein Feld sozialer Auseinandersetzungen darstellen, verstanden werden.

Die Überwindung des theoretischen Monismus ist daher eine wesentliche Voraussetzung, um überhaupt darüber nachdenken zu können, wie die Analyse der kapitalistischen Produktionsverhältnisse mit anderen gesellschaftlichen Formen und Achsen der Ungleichheit, Ausbeutung und Herrschaft, wie etwa den Geschlechterverhältnissen, Rassismus oder Heteronormativität vermittelt werden kann (Klinger 2007).

Auch die Dynamik und Krisenhaftigkeit kapitalistischer Gesellschaftsformationen und deren Bewältigung können aus dieser Perspektive nicht mehr als Entfaltung und Zuspitzung eines zentralen Widerspruchs, einer dominanten Dynamik aufgefasst werden, sondern erscheinen vielmehr als das konkrete und komplexe Zusammenspiel unterschiedlicher Entwicklungen und Prozesse und der damit verknüpften sozialen Kämpfe. Die vielfältigen Krisen und die Veränderungen der kapitalistischen Gesellschaftsformationen stellen daher keinen linearen und teleologischen (also beispielsweise auf eine finale Krise zusteuernden) Entwicklungsprozess dar. Sie sind vielmehr Gegenstand mannigfacher sozialer Auseinandersetzungen und Konflikte, deren Ergebnisse kontingent und offen sind.

Diese Überlegungen werfen natürlich auch Probleme für das Verständnis sozialer Kämpfe und der darüber konstituierten sozialen AkteurInnen auf. Diese wurden aber m.E. bis heute nicht auf befriedigende Weise gelöst. Vielmehr haben die Debatten um den Aufschwung rechtspopulistischer Mobilisierungen und deren Anspruch, sich als Bewegungen der unteren Schichten zu präsentieren und der sozialen Frage gegen die behaupteten Anmutungen des Feminismus, Antirassismus usw. wieder zu ihrem Recht zu verhelfen, die Frage nach den Verhältnissen zwischen den verschiedenen Ausbeutungs- und Herrschaftsverhältnissen (z. B. Klassen-, Geschlechter- und rassistische Verhältnisse), die kapitalistische Gesellschaftsformationen konstituieren, auf spezifische Weise aktualisiert. 
Die marxistischen und gesellschaftskritischen Debatten der letzten Jahrzehnte waren daher von der Frage geprägt, die Vervielfältigung der Subjektivierungsweisen und die Multiplikation der sozialen Kämpfe und Bewegungen theoretisch ernst zu nehmen, um daraus adäquate Vorstellungen zur Erneuerung gesellschaftspolitischer Transformationsperspektiven zu gewinnen. Die Analyse der Neuen sozialen Bewegungen, die Untersuchung der vielfältigen sozialen Kämpfe der Multitude, die Artikulation verschiedener Antagonismen in ein hegemoniales Projekt radikaler Demokratie etc. stellen daher Versuche dar, die Vielfalt der Emanzipationsperspektiven und sozialen Kämpfe mit dem Anspruch einer grundlegenden Veränderung kapitalistischer Gesellschaftsformationen zu verbinden (Hardt und Negri 2004; Aulenbacher 2013; Laclau und Mouffe 1991; Demirović 2011).

\subsection{Reproduktion durch Veränderung}

Die Fähigkeit des Kapitalismus, sich permanent zu verändern und Krisen durch die Transformation der Produktionsverhältnisse sowie der damit artikulierten gesellschaftlichen Institutionen zu bearbeiten, hat die Widersprüchlichkeit des marxistischen Verständnisses der Entwicklung der kapitalistischen Produktionsweise sichtbar gemacht. Einerseits hatten Marx und Engels bereits im kommunistischen Manifest die Dynamik des Kapitalismus auf eindrückliche Art und Weise dargestellt.

Alle festen eingerosteten Verhältnisse mit ihrem Gefolge von altehrwürdigen Vorstellungen und Anschauungen werden aufgelöst, alle neugebildeten veralten [...]. Alles Ständische und Stehende verdampft, alles Heilige wird entweiht, und die Menschen sind endlich gezwungen, ihre Lebensstellung, ihre gegenseitigen Beziehungen mit nüchternen Augen anzusehen. (Marx und Engels 1959, S. 465)

Und auch im „Kapital“ arbeitete Marx (1972, S. 511) heraus, dass die permanente Revolutionierung der Produktivkräfte eine wesentliche Grundlage der erweiterten Reproduktion der kapitalistischen Produktionsverhältnisse darstellt. Diese habe weitreichende Folgen für die jeweiligen Gesellschaftsformationen, da dadurch alle „Ruhe, Festigkeit, Sicherheit der Lebenslage“ (ebd.) der ArbeiterInnen und ihrer Familien aufgehoben werde.

Andererseits legt die marxsche Konzeptualisierung der Reproduktion der (kapitalistischen) Produktionsverhältnisse auch ein Verständnis nahe, wonach sie und die sie konstituierenden Stellungen in unveränderter Weise perpetuiert und gewissermaßen ,unbegrenzt konserviert“ (Balibar 2015, S. 535) werden. Reproduktion erscheint als automatisch ablaufender Prozess ohne Subjekt (ebd., S. 538).

Der kapitalistische Produktionsprozeß, im Zusammenhang betrachtet, oder als Reproduktionsprozeß, produziert also nicht nur Ware, nicht nur Mehrwert, er produziert und reproduziert das Kapitalverhältnis, auf der einen Seite den Kapitalisten, auf der anderen den Lohnarbeiter. (Marx 1972, S. 604)

Ob und inwieweit der „,beständige[...] Fluß [ihrer] Erneuerung“ (ebd., S. 591), auf den Marx im „Kapital“ verwiesen hatte, auch die Produktionsverhältnisse und die sie konstituierenden Agenten selbst verändert, wurde in marxistischen Analysestra- 
tegien, die etwa auf die behauptete Vertiefung der gesellschaftlichen Widersprüche abzielten, daher nicht ausreichend problematisiert.

Eine Dynamisierung des Reproduktionskonzeptes, das Veränderung als Bewegungsform der Reproduktion der kapitalistischen Gesellschaftsformationen sichtbar machen kann, ermöglicht es aber erstens, ihre Veränderungs- und Krisendynamiken zu analysieren, ohne diese in ein teleologisches Modell gesellschaftlicher Entwicklungen einzubetten. Vielmehr eröffnet sich dadurch die Frage, warum kapitalistische Gesellschaftsformationen trotz (oder wegen) ihrer grundlegenden Widersprüchlichkeit (Hirsch 2005; Aglietta 2000 [1979]) historisch unterscheidbare Modalitäten ihrer Reproduktion durch Veränderung stabilisieren können und welche Institutionen, gesellschaftlichen Verhältnisse oder AkteurInnen (Lipietz 1992, 1998) in die Regulation der kapitalistischen Produktionsverhältnisse und Akkumulationsprozesse einbezogen oder in sozialen Kämpfen neu gebildet werden.

Die krisenhafte Ablösung des fordistischen Entwicklungsmodells und der Übergang zum Finanzmarktkapitalismus können daher als Ablösung einer historisch spezifischen Transformationsweise kapitalistischer Gesellschaftsformationen und der konflikthaften Durchsetzung neuer Modi gesellschaftlicher Reproduktion durch Veränderung aufgefasst werden. Aus diesem Grund haben die Innovations- und Anpassungsfähigkeit der kapitalistischen Ökonomie und die damit verbundenen Auseinandersetzungen um die an den Verwertungsprozessen orientierte Reorganisation des gesellschaftlichen Institutionengefüges seit zwei bis drei Jahrzehnten massiv an Bedeutung gewonnen. Die forcierte Innovationsdynamik kapitalistischer Ökonomien stellt sich aus marxistischer Perspektive als spezifische Bewegungsform ihrer Widersprüche dar, die dazu führen, dass die sozialen Verhältnisse, die ihre TrägerInnen eingehen, und die sie bestimmenden Positionen und Qualitäten mit permanenten Veränderungserfordernissen konfrontiert sind.

In diesem Kontext wird daher zweitens eine zentrale Verschiebung sichtbar, die darauf hindeutet, dass die gegenwärtigen Formen der Revolutionierung der Produktivkräfte auf eine Transformation der sogenannten relativen Mehrwertproduktion ${ }^{6}$, wie sie Marx im „Kapital“ (1972) analysiert hatte, hinauslaufen. Die marxistischen Debatten des zwanzigsten Jahrhunderts (exemplarisch: Braverman 1977) hatten die aus der relativen Mehrwertproduktion folgenden Entwicklungstendenzen kapitalistischer Lohnarbeit als voranschreitende Entwertung und Dequalifikation der Ware Arbeitskraft, die die Einhegung und Kontrolle der (authentischen) Subjektivität sicherstellen sollten, analysiert. Der Übergang zum ,wissensbasierten“ (Jessop 2003) Kapitalismus in den Ländern des globalen Nordens hat dies aber auf grundlegende Art und Weise geändert. Nicht dass der Kapitalismus die Menschen daran hindert, ihre Anlagen und Fähigkeiten zu entwickeln, steht unter veränderten und sich verändernden Akkumulationsbedingungen im Zentrum, sondern dass sie gezwungen werden, diese im Sinne der ökonomischen Dynamik durch Lernen und andere Formen der Arbeit an ihrem Selbst permanent zu verändern und anzupassen. Die krisenhafte Transformation der Reproduktionsweise kapitalistischer Gesellschaftsformationen durch die permanente Revolutionierung der Produktivkräfte setzt die

\footnotetext{
${ }^{6}$ Bei der relativen Mehrwertproduktion geht es, vereinfacht gesprochen, um Steigerung des Mehrwerts durch Erhöhung der Produktivität der ArbeiterInnen mittels neuer Technologien.
} 
Subjekte und deren Arbeitsvermögen einem permanenten Veränderungsdruck aus. In diesen wird nicht zuletzt der Zugriff auf ihre Subjektivität zu einem zentralen Element der Krisenbearbeitung (Atzmüller 2015). Die Anpassungs-, Veränderungsund Lernfähigkeit des zum Humankapital transformierten Arbeitsvermögens werden zum Medium der Durchsetzung veränderter Formen der Krisenbearbeitung. Die marxistische Theoriebildung steht daher vor der Herausforderung, die veränderten Praxen gesellschaftlicher Subjektivierung im Kontext weitreichender Krisenprozesse und ihrer Bearbeitung zu konzeptualisieren, um die Veränderung der sozialen Widersprüche und Konfliktfelder zu identifizieren und existierende Konflikte auf ihren emanzipatorischen Gehalt hin untersuchen zu können. Die überkommenen Vorstellungen entfremdeter und entwerteter Subjektivität, die im marxistisch orientierten Verständnis aus den kapitalistischen Produktionsverhältnissen und den durch sie konstituierten Arbeitsprozessen resultieren, und ihre Befreiung durch eine revolutionäre Avantgarde greifen nicht mehr, da die Planung der eigenen Biographie und die Ausbildung, Entwicklung und Anpassung der Fähigkeiten zu Gestaltung und Steuerung der eigenen Subjektivität zur Anforderung und Zumutung für eine wachsende Zahl von Arbeitskräften geworden sind.

\section{Schlussfolgerungen}

Die skizzierten gesellschaftlichen wie auch epistemologischen Entwicklungen haben politische sowie theoretische und wissenschaftliche Vorstellungen, die auf Basis einer an die Wurzel und in diesem Sinne radikalen Kritik der Gesellschaft die Notwendigkeit, Wünschbarkeit und Möglichkeit grundlegender Veränderungen wissenschaftlich zu analysieren und begründen versuchen, in die Defensive gedrängt und ihre theoretischen Grundlagen und Annahmen fundamental erschüttert. Die marxistische Debatte steht daher vor der Frage, inwiefern die Veränderungsdynamik und Krisenhaftigkeit ihres Gegenstandes auch die erkenntnisgenerierende „Adäquatheit““ (Althusser et al. 2015) ihrer theoretischen Konzepte und Begriffe prekär werden ließ.

Dies ließ auch, wie analysiert, die gesellschaftskritische theoretische Praxis, die als zentrales Moment von Veränderungsprozessen zu verstehen ist, nicht unberührt. Durch die krisenhafte Transformation wurden überkommene Gewissheiten über die Zusammenhänge von Veränderung und Emanzipation prekär, da nun „Kontingenz“ (Demirović 2003) selbst zur zentralen Bewegungsform der Reproduktion der gesellschaftlichen Macht- und Herrschaftsverhältnisse wurde. Damit wird auch der Status der gesellschaftskritischen Theorie prekär, da sie aufgrund der Veränderungs- und Krisendynamik der realen gesellschaftlichen Prozesse ihren Status als systematische Erkenntnis der Wirklichkeit permanent zu verlieren und in eine verwirrende Vielfalt zu zerfallen droht. Die transformative Kapazität kritischer und insbesondere marxistischer Gesellschaftstheorien läuft dadurch Gefahr, permanent überholt zu werden. Man mag einwenden, dass das kein neues Problem sei, da es ein wesentliches Merkmal kapitalistischer Gesellschaftsformationen ist, dass sie sich durch Veränderung und Krisen reproduzieren. Das ändert aber nichts an der Herausforderung, dass marxistisch orientierte Theoriearbeit unter den gegenwärtigen Bedingungen ih- 
re begrifflichen und konzeptuellen Grundlagen einer eingehenden Überprüfung und gegebenenfalls Revision und Weiterentwicklung unterziehen muss.

Indeed, historical materialism is intrinsically a historical theory: its categories thus demand revision and development as new historical conditions and situations emerge. Revision is the very life of the Marxian dialectic, and the theory itself demands development, reconstruction, and even abandonment of obsolete or inadequate features as conditions emerge that put tenets of the original theory in question. (Kellner 1995, S. 34)

Funding Open access funding provided by Johannes Kepler University Linz.

Open Access Dieser Artikel wird unter der Creative Commons Namensnennung 4.0 International Lizenz (http://creativecommons.org/licenses/by/4.0/deed.de) veröffentlicht, welche die Nutzung, Vervielfältigung, Bearbeitung, Verbreitung und Wiedergabe in jeglichem Medium und Format erlaubt, sofern Sie den/die ursprünglichen Autor(en) und die Quelle ordnungsgemäß nennen, einen Link zur Creative Commons Lizenz beifügen und angeben, ob Änderungen vorgenommen wurden.

\section{Literatur}

Adorno, Theodor W. 2006. Spätkapitalismus oder Industriegesellschaft? In Die „Wissensgesellschaft“ Mythos, Ideologie oder Realität?, Hrsg. Uwe H. Bittlingmayer, Ullrich Bauer. Wiesbaden: VS.

Aglietta, Michel. 2000. A theory of capitalist regulation. The US experience. London, New York: Verso. Erstveröffentlichung 1979.

Althusser, Louis. 1978. Die Krise des Marxismus. Hamburg: VSA.

Althusser, Louis, Étienne Balibar, Roger Establet, Pierre Macherey, und Jacques Rancière (Hrsg.). 2015. Das Kapital lesen. Vollständige und ergänzte Ausgabe mit Retraktationen zum Kapital. Münster: Westfälisches Dampfboot.

Altvater, Elmar. 2010. Der große Krach oder die Jahrhundertkrise von Wirtschaft und Finanzen, von Politik und Natur. Münster: Westfälisches Dampfboot.

Altvater, Elmar, und Birgit Mahnkopf. 1996. Grenzen der Globalisierung - Ökonomie, Ökologie und Politik in der Weltgesellschaft, 1. Aufl., Münster: Westfälisches Dampfboot.

Altvater, Elmar, Hans-Jürgen Bieling, Alex Demirovic, Heiner Flassbeck, Werner Goldschmidt, Mehrdad Payandeh, und Stefanie Wöhl. 2010. Die Rückkehr des Staates? Nach der Finanzkrise. Hamburg: VSA.

Arrighi, Giovanni, und Jason W. Moore. 2001. Kapitalismus in welthistorischer Sicht. Das Argument 239:43-65.

Atzmüller, Roland. 2014. Aktivierung der Arbeit im Workfare-Staat. Arbeitsmarkt und Ausbildung nach dem Fordismus. Münster: Westfälisches Dampfboot.

Atzmüller, Roland. 2015. Transformation der ,zeitgemäßen Arbeitskraft“ und Krisenbearbeitung. In Die zeitgemäße Arbeitskraft. Qualifiziert, aktiviert, polarisiert, Hrsg. Roland Atzmüller, Stefanie Hürtgen, und Manfred Krenn, 195-310. Weinheim, Basel: Beltz Juventa.

Aulenbacher, Brigitte. 2013. Reproduktionskrise, Geschlechterverhältnis und Herrschaftswandel. Von der Frage nach Krisenherden über die Gesellschaftskritik zum Problem der Allianzen. In Krise, Kritik, Allianzen. Arbeits- und geschlechtersoziologische Perspektiven, Hrsg. Hildegard M. Nickel, Andreas Heilmann, 14-29. Weinheim: Beltz Juventa.

Aulenbacher, Brigitte, und Maria Dammayr (Hrsg.). 2014. Für sich und andere sorgen, Krise und Zukunft von Care in der modernen Gesellschaft. Weinheim, Basel: Beltz Juventa.

Balibar, Etienne. 2015. Die Grundbegriffe des historischen Materialismus. In Das Kapital lesen. Vollständige und ergänzte Ausgabe mit Retraktationen zum Kapital, Hrsg. Louis Althusser, Étienne Balibar, Roger Establet, Pierre Macherey, und Jacques Rancière, 441-592. Münster: Westfälisches Dampfboot.

Boltanski, Luc, und Ève Chiapello. 2006. Der neue Geist des Kapitalismus. Konstanz: UVK.

Boyer, Robert, und Yves Saillard (Hrsg.). 2002. Régulation theory. The state of the art. London, New York: Routledge.

Braverman, Harry. 1977. Die Arbeit im modernen Produktionsprozeß. Frankfurt a.M., New York: Campus. 
Bruff, Ian. 2013. The rise of authoritarian neoliberalism. Rethinking Marxism 26(1):113-129.

Davidson, Neil, und Richard Saull. 2017. Neoliberalism and the far-right. A contradictory embrace. Critical Sociology 43(4-5):707-724.

Demirovic, Alex, Sebastian Klauke, und Étienne Schneider (Hrsg.). 2015. Was ist der „Stand des Marxismus"? Soziale und epistemologische Bedingungen der kritischen Theorie heute. Münster: Westfälisches Dampfboot.

Demirović, Alex. 2003. Stroboskopischer Effekt und die Kontingenz der Geschichte. Gesellschaftstheoretische Rückfragen an die Regulationstheorie. In Fit für den Postfordismus? Theoretisch-politische Perspektiven des Regulationsansatzes, Hrsg. Ulrich Brand, Werner Raza, 43-57. Münster: Westfälisches Dampfboot.

Demirović, Alex. 2008. Leidenschaft und Wahrheit. Für einen neuen Modus der Kritik. In Kritik und Materialität, Hrsg. Alex Demirović, 9-40. Münster: Westfälisches Dampfboot.

Demirović, Alex. 2011. Kritische Gesellschaftsftheorie und die Vielfalt der Emanzipationsperspektiven. Prokla - Zeitschrift für kritische Sozialwissenschaft 41(164):519-542.

Demirović, Alex. 2015. Wissenschaft oder Dummheit? Über die Zerstörung der Rationalität in den Bildungsinstitutionen. Hamburg: VSA.

Demirović, Alex. 2018. Autoritärer Populismus als neoliberale Krisenbewältigungsstrategie. Prokla - Zeitschrift für kritische Sozialwissenschaft 48(1):27-42.

Demirović, Alex, Julia Dück, Florian Becker, und Pauline Bader (Hrsg.). 2011. Vielfachkrise im finanzdominierten Kapitalismus, in Kooperation mit dem wissenschaftichen Beirat von ATTAC. Hamburg: VSA.

Dörre, Klaus. 2009. Die neue Landnahme, Dynamiken und Grenzen des Finanzmarktkapitalismus. In Soziologie, Kapitalismus, Kritik. Eine Debatte, Hrsg. Klaus Dörre, Stephan Lessenich, und Hartmut Rosa, 21-86. Frankfurt a. Main: Suhrkamp.

Dörre, Klaus. 2013. Landnahme. Triebkräfte, Wirkungen und Grenzen kapitalistischer Wachstumsdynamik. In Die globale Einhegung - Krise, ursprüngliche Akkumulation und Landnahmen im Kapitalismus, Hrsg. Maria Backhouse, Olaf Gerlach, Stefan Kalmring, und Andreas Nowak, 112-140. Münster: Westfälisches Dampfboot.

Dörre, Klaus, Dieter Sauer, und Volker Wittke (Hrsg.). 2012. Kapitalismustheorie und Arbeit. Neue Ansätze soziologischer Kritik. Frankfurt a. Main, New York: Campus.

Freeman, Christopher, und Luc Soete. 1997. The economics of industrial innovation, 3. Aufl., London, Washington: Pinter.

Giddens, Anthony. 1978. The prospects for social theory today. Berkeley Journal of Sociology 23:201-223.

Gramsci, Antonio (Hrsg.). 1991. Gefängnishefte. Kritische Gesamtausgabe auf Grundlage der Edition von Valentino Gerratana. Hamburg: Argument.

Hall, Stuart. 1986. Popular-demokratischer und autoritärer Populismus. In Populismus und Aufklärung, Hrsg. Helmut Dubiel, 84-105. Frankfurt a. Main: Suhrkamp.

Hardt, Michael, und Antonio Negri. 2000. Empire. Cambridge: Harvard University Press.

Hardt, Michael, und Antonio Negri. 2004. Multitude. Krieg und Demokratie im Empire. Frankfurt a. M., New York: Campus.

Haug, Wolfgang F. 1996. Was kommt nach dem fordistischen Marxismus? Das Argument 214:183-199.

Haug, Wolfgang F. 2010. Krise des Marxismus. In Knechtschaft bis Krise des Marxismus, Hrsg. Wolfgang F. Haug, Frigga Haug, und Peter Jehle, 2160-2192.

Hirsch, Joachim. 1990. Kapitalismus ohne Alternative. Materialistische Gesellschaftstheorie und Möglichkeiten einer sozialistischen Politik heute. Hamburg: VSA.

Hirsch, Joachim. 2005. Materialistische Staatstheorie. Transformationsprozesse des kapitalistischen Staatensystems. Hamburg: VSA.

Jessop, Bob. 1990. State theory. Putting capitalist states in their place. Pennsylvania: Polity.

Jessop, Bob. 2003. Postfordismus und wissensbasierte Ökonomie. Eine Reinterpretation des Regulationsansatzes. In Fit für den Postfordismus? Theoretisch-politische Perspektiven des Regulationsansatzes, Hrsg. Ulrich Brand, Werner Raza, 89-111. Münster: Westfälisches Dampfboot.

Jessop, Bob. 2012. Narratives of crisis and crisis response: perspectives from north and south. In The global crisis and transformative social change, Hrsg. Shahra Razavi, Peter Utting, und Rebecca Varghese Buchholz, 23-42. Basingstoke: Palgrave Macmillan.

Jessop, Bob. 2015. The symptomatology of crisis, reading crisis and learning from them. Some criticial realist reflections. Journal of critical realism 14(3):238-271.

Jessop, Bob. 2016. Neoliberalismen, kritische politische Ökonomie und neoliberale Staaten. In Der Staat des Neoliberalismus, Hrsg. Thomas Biebricher, 123-152. Baden-Baden: Nomos. 
Kellner, Douglas. 1995. The end of orthodox Marxism. In Marxism in the postmodern age. Confronting the new world order, Hrsg. Antonio Callari, 33-41. Guilford: New York.

Klinger, Cornelia. 2007. Achsen der Ungleichheit. Zum Verhältnis von Klasse, Geschlecht und Ethnizität

Kouvélakis, Stathis. 2008. The crisis of marxism and the transformation of capitalism. In Critical companion to contemporary Marxism, Hrsg. Eustache Kouvélakis, Jacques Bidet, 23-38. Boston: Brill.

Krätke, Michael. 1996. Marxismus als Sozialwissenschaft. In Materialien zum Historisch-kritischen Wörterbuch des Marxismus. Für Wolfgang Fritz Haug zum 60. Geburtstag, Hrsg. Frigga Haug, 69-122. Hamburg: Argument.

Laclau, Ernesto, und Chantal Mouffe. 1991. Hegemonie und radikale Demokratie - Zur Dekonstruktion des Marxismus. Wien: Springer.

Lapavitsas, Costas. 2013. Profiting without producing. How finance exploits us all. London: Verso.

van der Linden, Marcel. 2009. Western Marxism and the Soviet Union. A survey of critical theories and debates since 1917. Chicago: Haymarket.

Lipietz, Alain. 1992. Vom Althusserismus zur „Theorie der Regulation“. In Hegemonie und Staat. Kapitalistische Regulation als Projekt und Prozeß, Hrsg. Alex Demirović, Hans-Peter Krebs, und Thomas Sablowski, 9-54. Münster: Westfälisches Dampfboot.

Lipietz, Alain. 1998. Nach dem Ende des „Goldenen Zeitalters“. Regulation und Transformation kapitalistischer Gesellschaften. Ausgewählte Schriften. Herausgegeben von Hans-Peter Krebs. Hamburg: Argument.

Marx, Karl. 1961a. Einleitung zur Kritik der Politischen Ökonomie. Werke 13., 615-641. Berlin: dietz.

Marx, Karl. 1961b. Kritik der Hegelschen Rechtsphilosophie. Werke 1., 378-391. Berlin: Dietz.

Marx, Karl. 1961c. Vorwort zur Kritik der politischen Ökonomie. Werke 13., 7-11. Berlin: dietz.

Marx, Karl. 1972. Das Kapital, Bd. 1. Werke 23. Berlin: Dietz.

Marx, Karl, und Friedrich Engels. 1959. Das Manifest der Kommunistischen Partei, MEW 4. Berlin: Dietz.

Perez, Carlota. 2012. Financial bubbles, crises and the role of government in unleashing golden ages

Streeck, Wolfgang. 2013. Gekaufte Zeit. Die vertagte Krise des demokratischen Kapitalismus. Frankfurter Adorno-Vorlesungen 2012. Frankfurt a. Main: Suhrkamp.

Sum, Ngai-Ling, und Bob Jessop. 2015. Towards a cultural political economy. Putting culture in its place in political economy. Cheltenham: Elgar.

Roland Atzmüller geb. 1969, Studium in Wien (Politikwissenschaft) und Manchester (GB). Zwischen 2002 und 2011 Mitarbeiter bei FORBA (Forschungs- und Beratungsstelle Arbeitswelt) in Wien. Seit 2011 am Institut für Soziologie der Johannes Kepler Universität Linz, Habilitation in Soziologie 2017. 